\title{
APPLICATION OF THE INTERVAL LATTICE BOLTZMANN METHOD FOR A NUMERICAL MODELING OF 2D THIN METAL FILMS IRRADIATED BY ULTRASHORT LASER PULSES
}

\author{
Alicja Piasecka-Belkhayat, Anna Korczak \\ Department of Computational Mechanics and Engineering \\ Silesian University of Technology, Gliwice, Poland \\ alicja.piasecka-belkhayat@polsl.pl,anna.korczak@polsl.pl
}

Received: 3 October 2019; Accepted: 20 December 2019

\begin{abstract}
In the paper, the two-dimensional numerical modelling of heat transfer in thin metal films irradiated by ultrashort laser pulses using the D2Q9 scheme is considered. In the mathematical description, the relaxation times and the boundary conditions for phonons and electrons are given as interval numbers. The problem has been formulated using the interval coupled lattice Boltzmann equations for electrons and phonons. The solution has been obtained by means of the interval lattice Boltzmann method using the rules of directed interval arithmetic. Examples of numerical computations are presented in the final part of the paper.
\end{abstract}

MSC 2010: 35Q2000, 35Q79

Keywords: lattice Boltzmann method, interval arithmetic, laser irradiation, heat transfer

\section{Introduction}

In metals, the heat transport is mainly realized by two kinds of heat carriers: electrons and phonons [1]. The electrons have higher velocities than phonons and by this way free electron mechanism of heat transport is much more efficient than the phonon one. Electrons and phonons always "move" from the part with the higher temperature to the part with the lower temperature and during this process electrons and phonons carry energy. This kind of phenomena can be described by the Boltzmann transport equation (BTE) [2-5]. The lattice Boltzmann method (LBM) is used to solve a discretized set of the Boltzmann transport equations [6]. In the paper, the coupled lattice Boltzmann equations for electrons and phonons have been assumed. The coupled model contains two energy equations determining the heat exchange in the electron gas and the metal lattice [7,8]. In general, for two-dimensional problems, D2Q4, D2Q5 and D2Q9 models are the models most common in the literature. In this paper the authors take into account D2Q9 model. 
Other approaches to the modeling of the microscale heat transfer are also used. Here, the different variants of the dual phase lag model e.g. [9-13] or the two-temperature parabolic or hyperbolic models e.g. [14-16] can be mentioned. It should be pointed out that in the case of metals and, under certain simplifications, the two-temperature parabolic model can be transformed to the dual phase one [17].

The heat transfer problems are usually solved using equations with deterministic parameters [1]. However, in most cases of the engineering practice, the values of these parameters cannot be defined with a high precision, and in such cases it is much more convenient to define these parameters as intervals numbers [7]. In the paper the interval values of relaxation times and the boundary conditions for electrons and phonons are taken into account. The relaxation times are estimated experimentally and their actual values are still a subject of discussion [18].

In the article, the authors present an innovative approach of the described problem using the interval lattice Boltzmann method with the approach of the directed interval arithmetic. In this arithmetic, two binary variables are defined: the direction variable and the sign variable. The set of all directed interval numbers can be defined as the sum of two sets: proper intervals and improper intervals, where the beginning of the interval is less than the end of the interval [19]. The main advantage of the directed interval arithmetic upon the usual interval arithmetic is that the obtained temperature intervals are much narrower and their width does not increase in time.

In theory, as well as in practice, it is valuable to develop the interval version of the LBM.

\section{The Boltzmann transport equation}

During the heating of thin metal films via laser pulse the electrons are energized and they subsequently transfer the energy to phonons via coupling between them. The Boltzmann transport equations for the coupled model (2D problem) with two kinds of carriers: electrons $(e)$ and phonons $(p h)$ transformed into carrier energy density equations take the following form [2]

$$
\begin{gathered}
\frac{\partial e_{e}}{\partial t}+\mathbf{v}_{e} \cdot \nabla e_{e}=\frac{e_{e}^{0}-e_{e}}{\tau_{e}}+Q_{e} \\
\frac{\partial e_{p h}}{\partial t}+\mathbf{v}_{p h} \cdot \nabla e_{p h}=\frac{e_{p h}{ }^{0}-e_{p h}}{\tau_{p h}}+Q_{p h}
\end{gathered}
$$

where $e_{e}, e_{p h}$ are the carrier energy densities, $e_{e}{ }^{0}, e_{p h}{ }^{0}$ are the equilibrium carrier energy densities and $Q_{e}, Q_{p h}$ are the carrier energy sources related to a unit of volume. The equations (1) and (2) must be supplemented by the adequate boundary-initial conditions. 
The electron and phonon energy densities at their equivalent nonequilibrium temperatures are given by the formulas

$$
\begin{gathered}
e_{e}\left(T_{e}\right)=\left(n_{e} \frac{\pi^{2}}{2} \frac{k_{b}^{2}}{\varepsilon_{F}}\right) T_{e}^{2} \\
e_{p h}\left(T_{p h}\right)=\left(\frac{9 \eta k_{b}}{\Theta_{D}^{3}} \int_{0}^{\Theta_{D} / T_{p h}} \frac{z^{3}}{\exp (z)-1} \mathrm{~d} z\right) T_{p h}{ }^{4}
\end{gathered}
$$

where $T_{e}, T_{p h}$ are the carrier temperatures, $k_{b}$ is the Boltzmann constant, $\varepsilon_{F}$ is the Fermi energy, $n_{e}$ is the electron density, $\Theta_{D}$ is the Debye temperature of the solid, $\eta$ is the number density of oscillators [2].

The electron and phonon energy sources are calculated using the following expressions [2]

$$
\begin{gathered}
Q_{e}=Q^{\prime}-G\left(T_{e}-T_{p h}\right) \\
Q_{p h}=G\left(T_{e}-T_{p h}\right)
\end{gathered}
$$

where $Q^{\prime}$ is the power density deposited by the external source function associated with the laser irradiation [18] and $G$ is the electron-phonon coupling factor which characterizes the energy exchange between electrons and phonons. The temporal variation of the laser output pulse is treated as a source term in the energy equation and may be approximated by a form of exponential function [19]

$$
Q^{\prime}(x, t)=I_{0} \delta e^{-\delta y-\beta t} e^{-\frac{2 x^{2}}{r^{2}}}
$$

where $I_{0}$ is the peak power intensity of the laser pulse, $\delta$ is the absorption coefficient, $\beta$ is the laser pulse parameter, $r$ is the radius of the laser beam, $x$ and $y$ are the coordinates. The laser parameter $\beta$ determines the laser pulse shape. Its value is in the range of $(0.5 \div 1) \cdot 10^{13} 1 / \mathrm{s}$.

\section{The interval lattice Boltzmann method}

The interval Boltzmann transport equations for the coupled model with two kinds of carriers ( $e$-electrons and $p h$-phonons) can be written using the following formulas $[2,20,21]$

$$
\frac{\partial \bar{e}_{e}}{\partial t}+v_{e x} \frac{\partial \bar{e}_{e}}{\partial x}+v_{e y} \frac{\partial \bar{e}_{e}}{\partial y}=-\frac{\bar{e}_{e}-\bar{e}_{e}^{0}}{\bar{\tau}_{r e}}+\bar{Q}_{e}
$$




$$
\frac{\partial \bar{e}_{p h}}{\partial t}+v_{p h x} \frac{\partial \bar{e}_{p h}}{\partial x}+v_{p h y} \frac{\partial \bar{e}_{p h}}{\partial y}=-\frac{\bar{e}_{p h}-\bar{e}_{p h}^{0}}{\bar{\tau}_{r p h}}+\bar{Q}_{p h}
$$

where $\bar{e}_{e}, \bar{e}_{p h}$ are the interval energy densities, $\bar{e}_{e}^{0}, \bar{e}_{p h}^{0}$ are the equilibrium interval energy densities, $\mathbf{v}_{e}=\left[v_{e x}, v_{e y}\right], \mathbf{v}_{p h}=\left[v_{p h x}, v_{p h y}\right]$ are the frequency-dependent propagation speed, $\bar{\tau}_{r e}, \bar{\tau}_{r p h}$ are the interval relaxation times, $t$ denotes the time and $\bar{Q}_{e}, \bar{Q}_{p h}$ are the interval energy sources related to a unit of volume for electrons and phonons respectively. For a two-dimensional 9-speed (D2Q9) model, the discrete carrier velocities $\left(\mathbf{v}_{d}=\mathbf{v}_{e}=\mathbf{v}_{p h}\right)$ are expressed as [13]

$$
\mathbf{v}_{d}=\left\{\begin{array}{cc}
(0,0), & d=0 \\
(\cos [(d-1) \pi / 2], \sin [(d-1) \pi / 2]), & d=1, \ldots, 4 \\
\sqrt{2}(\cos [(d-5) \pi / 2+\pi / 4], \sin [(d-5) \pi / 2+\pi / 4]) c, & d=5, \ldots, 8
\end{array}\right.
$$

where $c=\Delta x / \Delta t=\Delta y / \Delta t$ is the carriers speed, $\Delta x$ and $\Delta y$ are the lattice distances from site to site, $\Delta t=t^{f+1}-t^{f}$ is the time step needed for an electron or a phonon to travel from one lattice site to the neighboring lattice site and $d$ is the direction. For a 2D, model the discrete set of electron and phonon propagation velocities (10) in nine lattice directions might be also defined as (see Fig. 1)

$$
\begin{gathered}
\boldsymbol{v}_{e 0}=(0,0) \quad \boldsymbol{v}_{e 1}=(c, 0) \quad \boldsymbol{v}_{e 2}=(0, c) \quad \boldsymbol{v}_{e 3}=(-c, 0) \\
\boldsymbol{v}_{e 4}=(0,-c) \quad \boldsymbol{v}_{e 5}=(c, c) \quad \boldsymbol{v}_{e 6}=(-c, c) \quad \boldsymbol{v}_{e 7}=(-c,-c) \quad \boldsymbol{v}_{e 8}=(c,-c) \\
\boldsymbol{v}_{p h 0}=(0,0) \quad \boldsymbol{v}_{p h 1}=(c, 0) \quad \boldsymbol{v}_{p h 2}=(0, c) \quad \boldsymbol{v}_{p h 3}=(-c, 0) \\
\boldsymbol{v}_{p h 4}=(0,-c) \quad \boldsymbol{v}_{p h 5}=(c, c) \quad \boldsymbol{v}_{p h 6}=(-c, c) \quad \boldsymbol{v}_{p h 7}=(-c,-c) \quad \boldsymbol{v}_{p h 8}=(c,-c)
\end{gathered}
$$

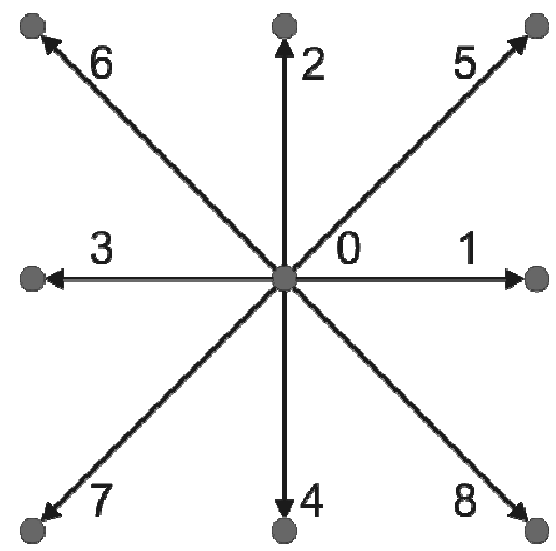

Fig. 1. Two-dimensional 9-speed lattice Boltzmann model (D2Q9) 


$$
\left\{\begin{aligned}
x=0,0 \leq y \leq L_{2}: \quad & \bar{q}_{b 1}^{e}(0, y, t)=c_{e}\left[\bar{e}_{e}\left(\bar{T}_{e}\right)_{0, \mathrm{j}}-\bar{e}_{e}\left(\bar{T}_{e}\right)_{1, \mathrm{j}}\right], \\
& \bar{q}_{b 1}^{p h}(0, y, t)=c_{p h}\left[\bar{e}_{p h}\left(\bar{T}_{p h}\right)_{0, \mathrm{j}}-\bar{e}_{p h}\left(\bar{T}_{p h}\right)_{1, \mathrm{j}}\right] ; j=0,1, \ldots, n \\
x=L_{1}, 0 \leq y \leq L_{2}: & \bar{q}_{b 2}^{e}\left(L_{1}, y, t\right)=c_{e}\left[\bar{e}_{e}\left(\bar{T}_{e}\right)_{\mathrm{m}, \mathrm{j}}-\bar{e}_{e}\left(\bar{T}_{e}\right)_{m-1, \mathrm{j}}\right], \\
& \bar{q}_{b 2}^{p h}\left(L_{1}, y, t\right)=c_{p h}\left[\bar{e}_{p h}\left(\bar{T}_{p h}\right)_{\mathrm{m}, \mathrm{j}}-\bar{e}_{p h}\left(\bar{T}_{p h}\right)_{m-1, \mathrm{j}}\right] ; j=0,1, \ldots, n \\
y=0,0<x<L_{1}: \quad & \bar{e}_{e}(x, 0, t)=\bar{e}_{e}\left(\bar{T}_{b 3}\right), \bar{e}_{p h}(x, 0, t)=\bar{e}_{p h}\left(\bar{T}_{b 3}\right) \\
y=L_{2}, 0<x<L_{1}: & \bar{q}_{b 4}^{e}\left(x, L_{2}, t\right)=c_{e}\left[\bar{e}_{e}\left(\bar{T}_{e}\right)_{i, n}-\bar{e}_{e}\left(\bar{T}_{e}\right)_{i, n-1}\right], \\
& \bar{q}_{b 4}^{p h}\left(x, L_{2}, t\right)=c_{p h}\left[\bar{e}_{p h}\left(\bar{T}_{p h}\right)_{i, n}-\bar{e}_{p h}\left(\bar{T}_{p h}\right)_{i, n-1}\right] ; i=0,1, \ldots, m
\end{aligned}\right.
$$

and the initial conditions

$$
\begin{array}{ll}
t=0: & e_{e}(x, y, 0)=e_{e}\left(T_{0}^{e}\right) \\
t=0: & e_{p h}(x, y, 0)=e_{p h}\left(T_{0}^{p h}\right)
\end{array}
$$

where $\bar{T}_{b 3}^{e}$ and $\bar{T}_{b 3}^{p h}$ are the interval values of boundary temperatures of electrons and phonons respectively, $\left(\bar{T}_{e}\right)_{i, n}$ and $\left(\bar{T}_{e}\right)_{i, n-1}$ are the interval temperatures of electrons on the top of the domain considered, $\left(\bar{T}_{p h}\right)_{i, n}$ and $\left(\bar{T}_{p h}\right)_{i, n-1}$ are the interval temperatures of phonons on the top of the domain considered, $T_{0}^{e}$ is the electron's initial temperature and $T_{0}{ }^{p h}$ is the phonon's initial temperature.

Taking into account formulas (10) and (11), a set of eighteen interval partial differential equations is obtained. Introducing discretizing form, time and position derivatives may be written as follows

$$
\begin{aligned}
& \left(\bar{e}_{e j}\right)_{i+1}^{f+1}=\left(1-\frac{\Delta t}{\bar{\tau}_{r e}}\right)\left(\bar{e}_{e j}\right)_{i}^{f}+\frac{\Delta t}{\bar{\tau}_{r e}}\left(\bar{e}_{e j}^{0}\right)_{i}^{f}+\Delta t\left[Q^{\prime}-G\left(\bar{T}_{e}^{f}-\bar{T}_{p h}^{f}\right)\right], j=0,1, \ldots, 8 \\
& \left(\bar{e}_{p h j}\right)_{i+1}^{f+1}=\left(1-\frac{\Delta t}{\bar{\tau}_{r p h}}\right)\left(\bar{e}_{p h j}\right)_{i}^{f}+\frac{\Delta t}{\bar{\tau}_{r p h}}\left(\bar{e}_{p h j}^{0}\right)_{i}^{f}+\Delta t G\left(\bar{T}_{e}^{f}-\bar{T}_{p h}^{f}\right), j=0,1, \ldots, 8
\end{aligned}
$$




\section{Results of computation}

In the final part of the paper the results of numerical computations are shown. As a numerical example, the heat transport in a gold thin film of the dimensions $1000 \mathrm{~nm} \times 200 \mathrm{~nm}$ has been analysed. To the modelling internal heat source were used parameters of the $\mathrm{KrF}$ laser: the wavelength of $\lambda=248 \mathrm{~nm}$ and the radius of the laser beam $r=42 \mathrm{~nm}$ [22]. The following input data have been introduced: the relaxation time $\bar{\tau}_{r e}=[0.038,0.042] \mathrm{ps}, \bar{\tau}_{r p h}=[0.76,0.84] \mathrm{ps}$, the Debye temperature $\Theta_{D}=170 \mathrm{~K}$, the peak power intensity of the laser pulse $I_{0}=2 \cdot 10^{13} \mathrm{~W} / \mathrm{m}^{2}$, the absorption coefficient $\delta=7.55 \cdot 10^{7} 1 / \mathrm{m}$, the laser pulse parameter $\beta=0.5 \cdot 10^{13} 1 / \mathrm{s}$, the coupling factor $\mathrm{G}=2.3 \cdot 10^{16} \mathrm{~W} / \mathrm{m}^{3} \mathrm{~K}$, the boundary conditions of the $1^{\text {st }}$ type on the bottom boundary with the interval temperature $\bar{T}_{b 3}^{e}=\bar{T}_{b 3}^{p h}=[285,315] \mathrm{K}$ and the $2^{\text {nd }}$ type on the other boundaries (where ' $\mathrm{k}$ ' means electron or phonon) $\bar{q}_{b 1}^{k}(0, y, t)=\bar{q}_{b 2}^{k}\left(L_{1}, y, t\right)=\bar{q}_{b 4}^{k}\left(x, L_{2}, t\right)=\overline{0}$, the the initial temperature $T_{0}=300 \mathrm{~K}$. The lattice step $\Delta x=20 \mathrm{~nm}$ and the time step $\Delta t=0.01 \mathrm{ps}$ have been assumed.

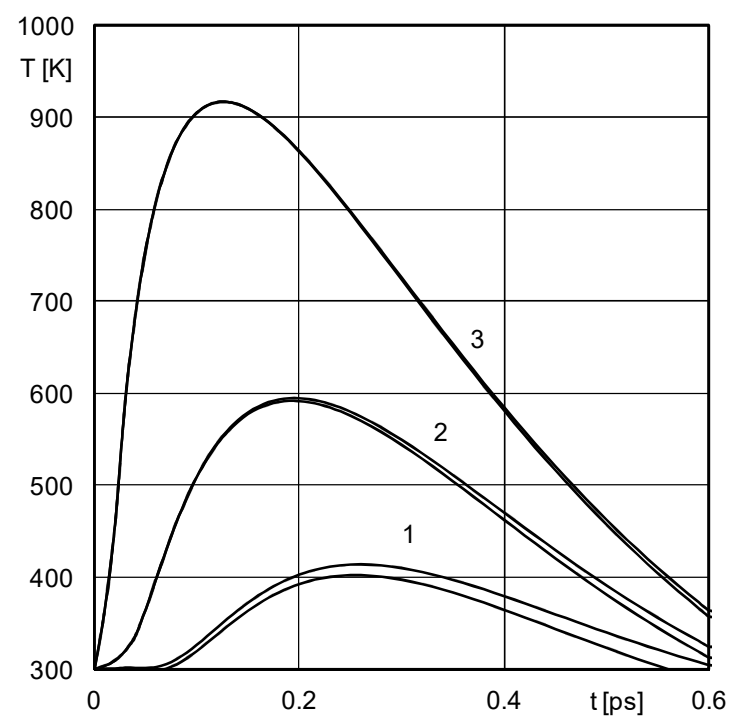

Fig. 2. The interval temperature distributions in gold film after $60 \mathrm{ps}$ for chosen nodes

Figure 2 illustrates the interval temperature distributions of electrons for interval parameters calculated for the nodes: $(140,60)-1,(140,100)-2,(140,140)-3$.

Figure 4 presents the interval temperature distributions of electrons for chosen times in the analysed domain (see Fig. 3). 


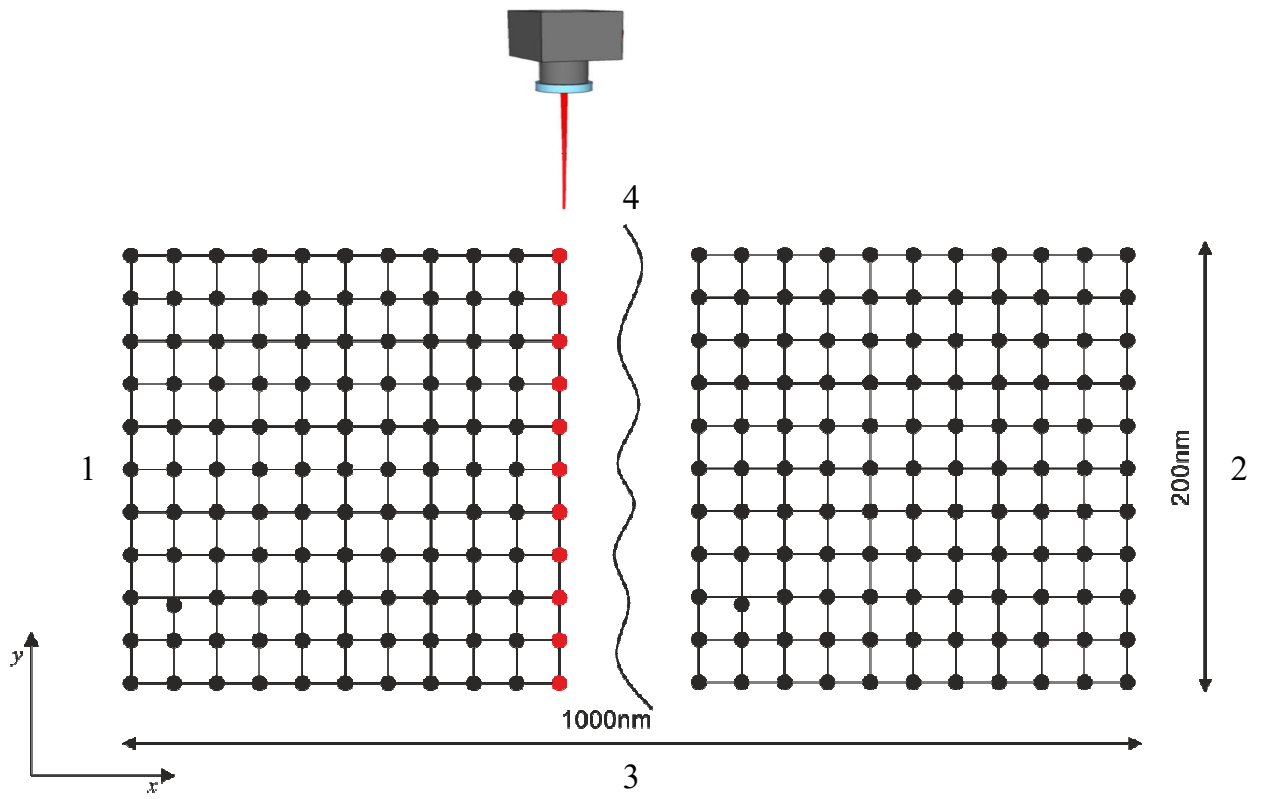

Fig. 3. Laser irradiation

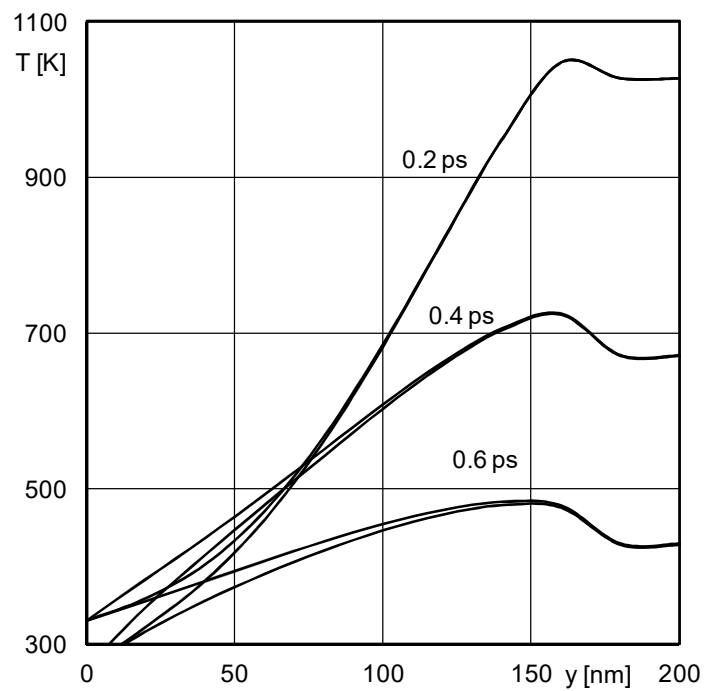

Fig. 4. The interval temperature distributions in gold film for chosen times

\section{Conclusions}

In the paper, the coupled lattice Boltzmann equations for electrons and phonons are applied to analyse the heating process of the thin metal films via laser pulse. The Boltzmann transport equations with the interval values of the relaxation times 
and the boundary conditions have been considered. The interval version of the lattice Boltzmann method for solving 2D problems has been presented using the rules of directed interval arithmetic. The generalization of LBM allows one to find the numerical solution in the interval form and such information may be important especially for the parameters that are estimated experimentally, for example the relaxation times.

\section{Acknowledgments}

The research is funded by the statutory grant of the Silesian University of Technology, Faculty of Mechanical Engineering, 2017.

\section{References}

[1] Eshraghi, M., \& Felicelli, S.D. (2012). An implicit lattice Boltzmann model for heat conduction with chase chandler. Int. J. of Heat and Mass Transfer, 55, 2420-2428.

[2] Escobar, R.A., \& Amon, C.H. (2008). Thin film phonon heat conduction by the dispersion lattice Boltzmann method. J. Heat Transfer, 130(9), 092402, 1-8.

[3] McNamara, G.R., \& Zanetti, G. (1988). Use of the Boltzmann equation to simulate lattice-gas automata. Phys. Rev. Lett., 61(20), 2332-2335.

[4] Higuera, F., Succi, S., \& Benzi, R. (1989). Lattice gas dynamics with enhanced collisions. Europhys. Let., 9(4), 345-349.

[5] Benzi, R., Succi, S., \& Vergassola, M. (1992). The lattice Boltzmann equation: theory and applications. Phys. Rep., 222(3), 145-197.

[6] Piasecka-Belkhayat, A. (2008). Interval boundary element method for 2D transient diffusion problem. Engineering Analysis with Boundary Elements, 32(5), 424-430.

[7] Chen, J.K., Tzou, D.Y., \& Beraun, J.E. (2006). A semiclassical two-temperature model for ultrafast laser heating. Int. J. of Heat and Mass Transfer, 49, 307-316.

[8] Joshi, A.A., \& Majumdar, A. (1993). Transient ballistic and diffusive phonon heat transport in thin films. J. of Appl. Physics, 74(1), 31-39.

[9] Zhang, Z.M. (2007). Nano/Microscale Heat Transfer. New York: McGraw-Hill.

[10] Tzou, D.Y. (2015). Macro- to Microscale Heat Transfer. The Lagging Behavior. New York: John Wiley\&Sons Ltd.

[11] Majchrzak, E., Mochnacki, B., \& Suchy J.S. (2009). Numerical simulation of thermal processes proceeding in a multi-layered film subjected to ultrafast laser heating. Journal of Theoretical and Applied Mechanics, 47, 2, 383-396.

[12] Deng, Y., Jiang, D., \& Liang, D. (2017). High-order finite difference method for a second order dual-phase-lagging models of microscale heat transfer. Applied Mathematics and Computations, 309, 31-48.

[13] Mochnacki, B., \& Paruch, M. (2013). Estimation of relaxation and thermalization time in microscale heat transfer model. $J$. of Theoretical and Applied Mechanics, 51, 4, 837-845.

[14] Al-Nimr, M.A. (1997). Heat transfer mechanisms during short duration laser heating of thin metal films. Int. J. Thermophys., 18, 5, 1257-1268.

[15] Lin, Z., \& Zhigilei, L.V. (2008). Electron-phonon coupling and electron heat capacity of metals under conditions of strong electron-phonon nonequilibrium. Phys. Rev. B, 77, 075133-1-075133-17. 
[16] Majchrzak, E., \& Dziatkiewicz, J. (2015). Analysis of ultashort laser pulse interactions with metal films using a two-temperature model. J. of Applied Mathematics and Computational Mechanics, 14(2), 31-39.

[17] Majchrzak, E., Mochnacki, B., Greer, A.L., \& Suchy, J.S. (2009). Numerical modeling of short pulse laser interactions with multi-layered thin metal films. CMES: Computer Modeling in Engineering and Sciences, 41, 2, 131-146.

[18] Lee, J.B., Kang, K., \& Lee, S.H. (2011). Comparison of theoretical models of electron-phonon coupling in thin gold films irradiated by femtosecond pulse lasers. Materials Transactions, 52(3), 547-553.

[19] Jasiński, M. (2015). Modelling of thermal damage in laser irradiated tissue. J. Appl. Math. Comput. Mech., 14, 67-78.

[20] Piasecka-Belkhayat, A. (2011). Interval boundary element method for imprecisely defined unsteady heat transfer problems. (in Polish), Monograph (321). Gliwice: Publ. of the Silesian University of Technology.

[21] Markov, S.M. (1995). On directed interval arithmetic and its applications. J. of Universal Comp. Science, 1, 514-526.

[22] Borovsky, A.V., Galkin, A.L., Shiryaev, O.B., \& Auguste, T. (2003). Laser Physics at Relativistic Intensities. Berlin: Springer. 